\title{
On connectivity of Julia sets of transcendental meromorphic maps and weakly repelling fixed points II
}

\author{
Núria Fagella Xavier JARQue Jordi TAIXÉS \\ Dept. de Matemàtica Aplicada i Anàlisi \\ Universitat de Barcelona \\ Gran Via, 585 - 08007 Barcelona
}

29th January 2010

\begin{abstract}
Following the attracting and preperiodic cases ([5]), in this paper we prove the existence of weakly repelling fixed points for transcendental meromorphic maps, provided that their Fatou set contains a multiply-connected parabolic basin. We use quasi-conformal surgery and virtually repelling fixed point techniques.
\end{abstract}

\section{Introduction}

Let $f$ be a rational, transcendental entire or transcendental meromorphic function. We say that a $p$-periodic point $z_{0}$ of $f$ is attracting (resp. repelling) if the modulus of its multiplier $\rho\left(z_{0}\right):=\left(f^{p}\right)^{\prime}\left(z_{0}\right) \in \mathbb{C}$ is smaller (resp. greater) than 1 , and that it is parabolic if $\rho\left(z_{0}\right)=e^{2 \pi i \theta}$, with $\theta \in \mathbb{Q}$. Furthermore, $z_{0}$ is said to be weakly repelling if it is repelling or parabolic of multiplier 1. For any rational map of degree greater than one, the existence of at least one such fixed point is guaranteed by a theorem of Fatou [6].

As for global dynamics, points can be classified according to their longterm behaviour under iteration of the function, thus one defines the Fatou set $\mathcal{F}(f)$ (or simply $\mathcal{F}$ when possible) as the set of points $z_{0} \in \widehat{\mathbb{C}}$ for which the family $\left\{f^{k}\right\}_{k \in \mathbb{N}}$ is defined and normal in a neighbourhood of $z_{0}$, and the Julia set as its complement, $\mathcal{J}=\mathcal{J}(f):=\widehat{\mathbb{C}} \backslash \mathcal{F}(f)$. Then a connected component of the Fatou set (Fatou component) $U$ is called preperiodic if there are integers $p>q \geq 0$ such that $f^{p}(U)=f^{q}(U)$, and, more precisely,

All authors were supported by the Spanish grant MTM2008-01486, the Catalan grant 2009SGR-00792 and European funds MCRTN-CT-2006-035651. Also, the first and the second authors were supported by MTM2006-05849/Consolider (including a FEDER contribution) and the third author by $2009 \mathrm{CTP}-00002$. 
$p$-periodic when $q=0$ and fixed when, moreover, $p=1$. On the contrary, a Fatou component is called a wandering domain if it fails to be preperiodic.

According to the work of Cremer and Fatou, a $p$-periodic Fatou component $U$ is necessarily one of the following: immediate attractive basin, if $U$ contains an attracting $p$-periodic point $z_{0}$ such that $\lim _{n \rightarrow \infty} f^{n p}(z)=z_{0}$ for all $z \in U$; parabolic basin or Leau domain, if $\partial U$ contains a $q$-periodic point $z_{0}$, with $q \mid p$, such that $\lim _{n \rightarrow \infty} f^{n q}(z)=z_{0}$ for all $z \in U$ (and $\left(f^{p}\right)^{\prime}\left(z_{0}\right)=1$ ); Siegel disc, if there exists a holomorphic homeomorphism $\phi: U \rightarrow \mathbb{D}$ such that $\left(\phi \circ f^{p} \circ \phi^{-1}\right)(z)=e^{2 \pi i \theta} z$ for some $\theta \in \mathbb{R} \backslash \mathbb{Q} ;$ Herman ring, if there exist an $r>1$ and a holomorphic homeomorphism $\phi: U \rightarrow\{1<|z|<r\}$ such that $\left(\phi \circ f^{p} \circ \phi^{-1}\right)(z)=e^{2 \pi i \theta} z$ for some $\theta \in \mathbb{R} \backslash \mathbb{Q} ;$ Baker domain, if $\partial U$ contains a point $z_{0}$, with $f^{p}\left(z_{0}\right)$ not defined, such that $\lim _{n \rightarrow \infty} f^{n p}(z)=z_{0}$ for all $z \in U$. Nevertheless, rational maps happen to have neither wandering domains (Sullivan [11]) nor Baker domains (for infinity is but a regular point there).

With this setting, we are now able to describe the purpose and results of this paper. In 1990, Shishikura [10] proved that if the Julia set of a rational map $f$ is disconnected, then $f$ has at least two weakly repelling fixed points. As a consequence, the connectedness of the Julia set of the Newton's method $N_{f}:=\mathrm{id}-f / f^{\prime}$ for a non-constant polynomial $f$ is obtained forthwithsince all its fixed points but infinity are attracting - , hence closing a problem of which Przytycki [9], Meier [7] and Tan Lei [12] among others had given partial results before.

As pointed out in [5], our purpose is to give the natural transcendental versions of Shishikura's results, namely: If the Julia set of a transcendental meromorphic function $f$ is disconnected, then $f$ has at least one weakly repelling fixed point; the connectedness of the Julia set of the Newton's method for transcendental entire functions would follow as a corollary. Notice the equivalence between such hypothesis and at least one of the Fatou components of $f$ being multiply connected (as opposed to simply connected), which allows each different type of Fatou component to be treated separately.

In [5] we proved the following result, which covers the immediate attractive basin and preperiodic Fatou component cases.

Theorem A. Let $f$ be a transcendental meromorphic function with either a multiply-connected immediate attractive basin or a multiply-connected Fatou component $U$ such that $f(U)$ is simply connected. Then, there exists at least one weakly repelling fixed point of $f$.

On the other hand, the case of the multiply-connected wandering domain was proved by Bergweiler and Terglane in the search of solutions of certain differential equations with no wandering domains (see [2]). Now the present work is devoted to parabolic basins. More precisely, we prove the following. 
Theorem B. Let $f$ be a transcendental meromorphic function with a multiply-connected parabolic basin. Then $f$ has at least one weakly repelling fixed point.

The main tools involved in its proof are the method of quasi-conformal surgery and a theorem of Buff on virtually repelling fixed points; we shall briefly introduce such two concepts in Section 2. Then Section 3 contains the actual proof for Theorem B.

\section{The tools}

\subsection{Quasi-conformal surgery}

Quasi-conformal surgery is a powerful technique that derives from an analytical study of quasi-conformal maps and has many applications in complex dynamics, since it can produce holomorphic maps with some prescribed dynamics. In a first step, known as topological surgery, one takes a number of spaces and functions having a certain behaviour locally or in some suitable subspace. Roughly speaking, these can be cut and assembled together so as to construct a map with the dynamics chosen a priori. Now the measurable Riemann mapping theorem (Theorem 2.1) can be applied to such map in order to make it holomorphic via a quasi-conformal conjugation. This second step of the process is usually called holomorphic smoothing.

The following are just a few necessary definitions and results for the surgery process to be completed. See for example [4] for a more comprehensive text on quasi-conformal surgery.

Definition. Let $U \subset \mathbb{C}$ be an open set; a measurable function $\mu: U \rightarrow \mathbb{C}$ is called a $k$-Beltrami coefficient of $U$ if $\|\mu\|_{\infty}=k<1$.

Equivalently, one can associate to every $k$-Beltrami coefficient of $U \mu$ an almost complex structure $\sigma$, that is, a measurable field of (infinitesimal) ellipses in $T U$, defined up to multiplication by a positive real constant. More precisely, the argument of the minor axis of such ellipses at a point $z \in U$ is $\arg (\mu(z)) / 2$, and its ellipticity - i.e. the ratio between its axesequals $(1-|\mu(z)|) /(1+|\mu(z)|)$. Notice that this value is bounded between $\left(1-\|\mu\|_{\infty}\right) /\left(1+\|\mu\|_{\infty}\right)>0$ and 1 almost everywhere.

Definition. Let $U$ and $V$ be open sets in $\mathbb{C}$; a homeomorphism $\phi: U \rightarrow$ $V$ is said to be $k$-quasi-conformal if it has locally square integrable weak derivatives (and therefore it is differentiable almost everywhere) and the function

$$
\mu_{\phi}(z):=\frac{\partial \phi / \partial \bar{z}}{\partial \phi / \partial z}(z)
$$


defined almost everywhere in $U$, is a $k$-Beltrami coefficient. A $k$-quasiregular map is the composition of a holomorphic function and a quasiconformal map, or, equivalently, a map which is locally quasi-conformal around every point except for a discrete set of points - the critical points.

Definition. Let $U$ and $V$ be open sets in $\mathbb{C}$; a quasi-regular map $\phi: U \rightarrow V$ induces a contravariant functor $\phi^{*}: L^{\infty}(V) \rightarrow L^{\infty}(U)$ defined by

$$
\phi^{*} \mu:=\frac{\partial \phi / \partial \bar{z}+(\mu \circ \phi)(\overline{\partial \phi / \partial z})}{\partial \phi / \partial z+(\mu \circ \phi)(\overline{\partial \phi / \partial \bar{z}})} .
$$

Notice that if $\mu: V \rightarrow \mathbb{C}$ is a Beltrami coefficient, then so is its pull-back $\phi^{*} \mu: U \rightarrow \mathbb{C}$. Moreover, if $\phi$ is a holomorphic map, then $\left\|\phi^{*} \mu\right\|_{\infty}=\|\mu\|_{\infty}$.

When the Beltrami coefficient $\mu$ is defined in terms of a quasi-regular map $\psi$ as above $\left(\mu \equiv \mu_{\psi}\right)$, one can check that $\phi^{*} \mu_{\psi}=\mu_{\psi \circ \phi}$.

Definition. We call standard complex structure the constant Beltrami coefficient $\mu_{0}:=0$ or, equivalently, the associated field of circles $\sigma_{0}$.

By Weyl's Lemma, we have that a quasi-regular map $\phi$ is holomorphic if, and only if, $\phi^{*} \mu_{0}=\mu_{0}$.

Now, it is clear that a quasi-conformal map $\phi$ defines a Beltrami coefficient $\mu_{\phi}$. Conversely, given a Beltrami coefficient $\mu$ and the so-called Beltrami equation

$$
\frac{\partial \phi}{\partial \bar{z}}=\mu \cdot \frac{\partial \phi}{\partial z},
$$

can we find an actual quasi-conformal map $\phi$ such that $\mu_{\phi} \equiv \mu$ (equivalently, $\left.\phi^{*} \mu_{0}=\mu\right)$ ? The celebrated measurable Riemann mapping theorem answers this question positively; the following is a version of the statement with $U=V=\mathbb{C}$ (see also [1] or [4]).

Theorem 2.1 (Morrey, Bojarski, Ahlfors, Bers). Let $\mu$ be a Beltrami coefficient of $\mathbb{C}$; then, there exists a unique (up to postcomposition with conformal maps of $\mathbb{C})$ quasi-conformal map $\phi: \mathbb{C} \rightarrow \mathbb{C}$ such that $\partial \phi / \partial \bar{z}=\mu \cdot \partial \phi / \partial z$ (or $\left.\mu_{\phi}=\mu\right), \phi(0)=0$ and $\phi(1)=1$.

The application of this result to complex dynamics is the following. Suppose that $f: \widehat{\mathbb{C}} \rightarrow \widehat{\mathbb{C}}$ is a quasi-regular map whose dynamics we would like to see realised by a holomorphic map of $\widehat{\mathbb{C}}$. Then, Theorem 2.1 guarantees the existence of such a map as long as we can construct an appropriate $f$ invariant almost complex structure. The precise statement reads as follows.

Corollary 2.2. Let $\mu$ be a Beltrami coefficient of $\mathbb{C}$ and $f: \widehat{\mathbb{C}} \rightarrow \widehat{\mathbb{C}}$ a quasiregular map such that $f^{*} \mu=\mu$; then, $f$ is quasi-conformally conjugate to a holomorphic map $g: \widehat{\mathbb{C}} \rightarrow \widehat{\mathbb{C}}$. 
Proof. Applying the measurable Riemann mapping theorem to $\mu$, there exists a quasi-conformal map $\phi$ with $\mu=\phi^{*} \mu_{0}$. Now, let us define $g:=$ $\phi \circ f \circ \phi^{-1}$; we just need to see that $g$ is indeed holomorphic:

$$
g^{*} \mu_{0}=\left(\phi f \phi^{-1}\right)^{*} \mu_{0}=\left(\phi^{-1}\right)^{*} f^{*} \phi^{*} \mu_{0}=\left(\phi^{-1}\right)^{*} f^{*} \mu=\left(\phi^{-1}\right)^{*} \mu=\mu_{0} .
$$

\subsection{On virtually repelling fixed points}

In the search of a weakly repelling fixed point, notice that the construction of a polynomial-like map situation would indeed suffice. However, such construction may not always be totally straightforward - or even possible because of the necessity to find a domain of definition of the map strictly contained inside its own image. Nevertheless, a theorem of Buff (see [3]) gives so-called virtually repelling fixed points under slightly milder hypothesis. The key point about working with fixed points that are virtually repelling instead of weakly repelling is that the first condition is slightly stronger than the second one - and Theorem 2.3 provides them.

In what follows we define this concept and state Buff's theorem.

Definition. The holomorphic index of a complex function $f$ at a fixed point $z$ is the residue

$$
\iota(f, z):=\frac{1}{2 \pi i} \oint_{z} \frac{d w}{w-f(w)} .
$$

In the case of a simple fixed point (i.e., with multiplier $\rho(z) \neq 1$ ), the index is also given by

$$
\iota(f, z)=\frac{1}{1-\rho(z)} .
$$

If we have that $\operatorname{Re}(\iota(f, z))<m / 2$, where $m \geq 1$ denotes the multiplicity, the fixed point $z$ is called virtually repelling.

Theorem 2.3 (Buff). Let $U \subset \mathbb{D}$ be an open set and $f: U \rightarrow \mathbb{D}$ a proper holomorphic map of degree $d \geq 2$. If $|f(z)-z|$ is bounded away from zero as $z \in U$ tends to $\partial U$, then $f$ has at least one virtually repelling fixed point. form.

Using the Riemann map, the theorem can be stated in a more general

Corollary 2.4. Let $V$ and $D$ be open sets of $\mathbb{C}$ with $V \subset D$ and $D$ simply connected, and let $f: V \rightarrow D$ be a proper holomorphic function. If $|f(z)-z|$ is bounded away from zero as $z \in V$ tends to $\partial V$, there exists at least one virtually repelling fixed point of $f$. 




Figure 1: Sketch of the proof of Corollary 2.4. Notice that $D$ or $V$ might be unbounded, and that $V$ has no topological conditions other than being open, so, in particular, it need not be simply connected. This prevents us from using an argument of polynomial-like mappings to find a weakly repelling fixed point, even if $\partial V \cap \partial D=\emptyset$.

Proof. Since the set $D$ is open and simply connected, we have that there exists a conformal Riemann mapping $\varphi: D \rightarrow \mathbb{D}$. This map takes the subset $V$ to some $\varphi(V)=U \subset \mathbb{D}$, as $V$ is contained in $D$. (See Figure 1.)

Let us now define the map $g:=\varphi \circ f \circ \varphi^{-1}$, which is conjugate to $f$ by the conformal conjugation $\varphi$. Observe that $g$ is proper and $|g(z)-z|$ is bounded away from zero as $z \in U$ tends to $\partial U$. In this situation, $g$ has at least one virtually repelling fixed point $z_{0}$ due to Theorem 2.3. Since conformal conjugacies preserve this property of fixed points, we have that there exists a virtually repelling fixed point $\varphi^{-1}\left(z_{0}\right)$ of $f$ (in $V$ ).

Recall that a map $f: X \rightarrow Y$ is proper if the preimage set $f^{-1}(K) \subset$ $X$ is compact for any compact set $K \subset Y$. Although our transcendental meromorphic maps are of infinite degree and may not be proper globally, Corollary 2.4 can still be applied to subsets $V \subset \widehat{\mathbb{C}}$ where the restriction $\left.f\right|_{V}$ be proper and hence of finite degree, as the following lemma shows.

Lemma 2.5. Let $f$ be a transcendental meromorphic function, $Y \subset \mathbb{C}$ an open, simply-connected set and $X$ a bounded connected component of $f^{-1}(Y)$. Then, the restriction $\left.f\right|_{X}: X \rightarrow Y$ is a proper map.

Proof. First notice that $\left.f\right|_{X}$ is of finite degree because $X$ is bounded. Now, let $K$ be a compact set of $Y$; it follows that $\infty \notin K$. Also, $f^{-1}(K) \subset X$ is bounded, so $f$ is locally $\left(\left.f\right|_{f^{-1}(K)}: f^{-1}(K) \rightarrow K\right)$ holomorphic and, therefore, the preimage set $f^{-1}(K)$ of the compact set $K \subset Y$ is also compact. 


\section{Multiply-connected parabolic basin}

In this section we prove Theorem B (see Section 1). Its proof involves two different techniques. The first one is based upon Shishikura's proof and applies when preimages of certain sets do not behave too wildly in the presence of the essential singularity. For the second one, the assumption of a pole of $f$ allows us to construct some sets where the hypothesis of Corollary 2.2 are met.

Recall that by $p$-periodic parabolic basin $B$ we understand a connected component of the Fatou set such that there exists a $q$-periodic point $\alpha \in \partial B$, $q \mid p$, with $\lim _{n \rightarrow \infty} f^{n q}(z)=\alpha$ for all $z \in B$ and, in particular, $\left(f^{p}\right)^{\prime}(\alpha)=$ 1 (i.e., the immediate basin associated to a one petal attached to a $q$ periodic parabolic point). Notice that $p$ is the period of $B$, not of $\alpha$, so $B, f(B), \ldots, f^{p-1}(B)$ are pairwise disjoint. Also, $p / q$ gives the number of petals sharing $\alpha$ as a boundary point.

The rest of the section is the proof of Theorem B.

First notice that if $p=1$ (and so $q=1$ ) then there exists a fixed point $\alpha \in \partial B$ such that $f^{\prime}(\alpha)=1$, i.e., there exists a weakly repelling fixed point of $f$ and we are done. So let us assume from now on that $p>1$.

Let $\langle\alpha\rangle$ be the cycle of points generated by the iteration of the $q$-periodic parabolic point $\alpha$. We want to construct a sequence of open sets $\left\{U_{k}\right\}_{k}$, starting with a simply connected one, such that $\langle\alpha\rangle \cap \partial U_{k} \neq \emptyset$ and $f\left(U_{k+1}\right)=$ $U_{k}$ for all $k \geq 0$.

In the following we use the so-called Fatou coordinates, see e.g. [8]. Without loss of generality we can assume that $\alpha=0$ by a coordinate change, and $f^{p}$ to be in normal form $f^{p}(z)=z\left(1+a z^{\nu}+\mathcal{O}\left(z^{\nu+1}\right)\right)$, for some $a \in \mathbb{C}$ and $\nu=p / q$. Let $U_{0} \subset B$ be the pull-back $U_{0}:=H^{-1}(\{w: \operatorname{Re} w>L\})$, where $H(z):=-1 / \nu a z^{\nu}$ and $L>0$ is large and to be precised later. It is easy to check that $H$ is an actual conjugacy between $f^{p}$ and

$$
T(w):=\left(H \circ f^{p} \circ H^{-1}\right)(w)=w+1+\mathcal{O}\left(w^{-1 / \nu}\right),
$$

hence we can choose $L$ large enough so that $f^{p}$ is injective on $U_{0}$ (see Figure 2 ). Also, notice that $f^{p}\left(\overline{U_{0}}\right) \subset U_{0} \cup\{\alpha\}$ because of the action of $T$.

Now define $\left\{U_{k}\right\}_{k \in \mathbb{N}}$ by pulling back $U_{0}$ under $f$, namely, $U_{k}$ is the connected component of $f^{-k}\left(U_{0}\right)$ such that $\partial U_{k} \cap\langle\alpha\rangle \neq \emptyset$. Notice that $U_{j} \subset U_{p+j} \subset U_{2 p+j} \subset \ldots$ and $f^{j}(B)=\bigcup_{k \geq 1} U_{k p-j}$, for all $0 \leq j<p$. Because $B$ is multiply connected, there exists a (minimal) $n_{0} \in \mathbb{N}$ such that $U:=U_{n_{0}}$ is also multiply connected. Call $E$ one of the bounded connected components of $\widehat{\mathbb{C}} \backslash U$. Notice that $E$ is compact and full, and $\stackrel{\circ}{E}$ need not be connected.

Now preimages of compact sets under transcendental meromorphic maps might become unbounded and eventually contain poles and prepoles. This fact will be an obstacle to follow Shishikura's proof of the rational case, as 




Figure 2: Construction of $U_{0}$ as an $H$-pull-back of the half-plane $\{w: \operatorname{Re} w>L\}$ (example with $\nu=3$, so $p=3 q$ ). Notice that $\overline{U_{0}}$ contains no critical points, since $\left.f^{p}\right|_{U_{0}}$ is injective. Furthermore, we can choose $L$ in such a way that $\partial U$ meets not the postcritical set (orbits of the critical points). because critical points can only accumulate at an essential singularity.

we will show later; so, at this point, we split the proof according to the nature of $\partial E$.

Case 1: $\partial E$ contains at least one pole

If $\partial E$ contains at least one pole $P$, then $f(U)$ is unbounded (since $\partial E \subset \partial U$ ) and so is contained in some unbounded connected component of $\widehat{\mathbb{C}} \backslash U$ (since $p>1$ and therefore $U \cap f(U)=\emptyset$ ). Let $V \subset \widehat{\mathbb{C}}$ be a connected simplyconnected unbounded open set such that $U \subset V$ and $f(U) \subset \widehat{\mathbb{C}} \backslash \bar{V}$, for example, $V$ could be the connected component of $\widehat{\mathbb{C}} \backslash \overline{f(U)}$ containing $E$. (Notice that $E \subset V$, for $V$ is simply connected and $E$ is bounded.) Now there exists a connected component $D$ of $f^{-1}(V)$ such that $P \in \partial D$, and $D \subset E$ since points immediately outside $E$ happen to be in $U$, thus mapped into $f(U) \subset \mathbb{\mathbb { C }} \backslash V$ (see Figure 3). Notice that Lemma 2.2 gives the properness of $\left.f\right|_{D}: D \rightarrow V$, since $D \subset E$ is bounded and $V$ simply connected. Moreover, $D \subset E \subset V$, so $\partial D \cap \partial V=\emptyset$ and we can apply Buff's theorem (and Corollary 2.4 ) to find a weakly repelling fixed point of $f$.

Case 2: $\partial E$ contains no poles

Now if $\partial E$ contains no poles, $f(U)$ is bounded (and simply connected by construction) therefore no other component of $\widehat{\mathbb{C}} \backslash U$ can have poles on its boundary. (Still, further images of $U$ might be unbounded, for example, if $\partial E$ contains prepoles.) Let us assume, without loss of generality, that $f(U), \ldots, f^{k-1}(U) \subset E$ and $f^{k}(U) \subset \widehat{\mathbb{C}} \backslash E$, for some $1 \leq k \leq p$. In that case we will use the quasi-conformal surgery technique, but must be careful with the set of preimages of $\alpha$, that might intersect $\partial U$ and make the whole 

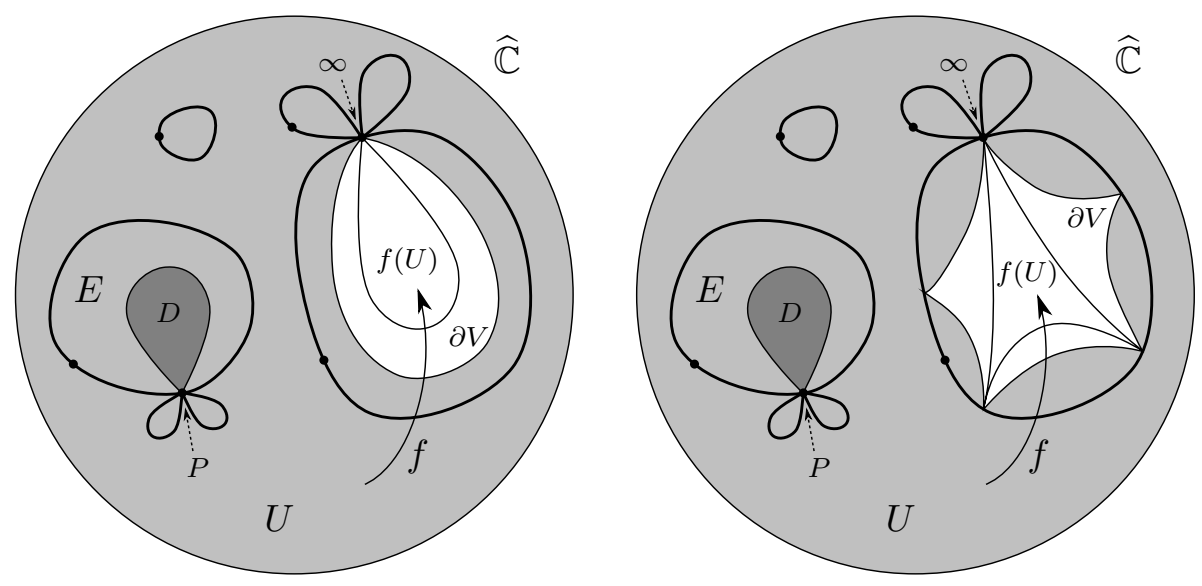

Figure 3: If there exists a pole $P$ on $\partial E$, then there exists a set $D \subset E$ such that $f(D)=V$, where $V$ is an unbounded simply-connected set that contains $U$ but not $f(U)$. The thick lines correspond to $\partial U$, while the sets $D$ and $V$ appear dark- and light-shaded, respectively. The non-labelled points represent the different places where $\alpha$ can lie. On the right, a case where $\partial U \cap \partial f(U) \neq \emptyset$.

process somewhat laborious.

In fact, a key point during the surgery process is the construction of an interpolating map between two different functions on two disjoint closed curves. If such curves are to touch at preimages of $\alpha$ or at $\alpha$ itself, this interpolation cannot be performed and an extra step previous to surgery will be done. Since we are focusing our attention on boundary intersections here, we shall still resplit this case into two finer subcases as follows.

Case 2.1: $k<p$, or $k=p$ but $\partial f^{p}(U) \cap \partial E=\emptyset$

First notice that if $k<p$ then $\partial f^{k}(U) \cap \partial E=\emptyset: f^{k}(U)$ cannot be contained in $U$ because $f^{p}(U)$ is the first iteration back inside $U$, by construction; therefore $f^{k}(U)$ must be contained in a component of $\widehat{\mathbb{C}} \backslash U$ other than $E$, hence $\partial f^{k}(U) \cap \partial E=\emptyset$ since two different components of $\widehat{\mathbb{C}} \backslash U$ cannot form a connected set. Then, we can apply quasi-conformal surgery as follows: Define a quasi-regular map $f_{2}: \widehat{\mathbb{C}} \rightarrow \widehat{\mathbb{C}}$ that will map $\widehat{\mathbb{C}} \backslash E$ strictly inside itself (after $k$ iterations). More precisely, set $V_{0}:=\widehat{\mathbb{C}} \backslash E$ and $V_{1}:=f(U)$, which lies in either $E$ (when $k>1$ ) or $\widehat{\mathbb{C}} \backslash E$ (when $k=1$ ). Set also $K:=\overline{f^{k}(U)}$ and choose $b \in f(U)$ and $a=f^{k-1}(b) \in K$ (see Figure 4).

Lemma 3.1 (Interpolation Lemma). Let $V_{0}$ and $V_{1}$ be simply-connected open sets in $\widehat{\mathbb{C}}$, with $V_{0} \neq \widehat{\mathbb{C}}$, and $f$ a holomorphic map from a neighbourhood $N$ of $\partial V_{0}$ to $\widehat{\mathbb{C}}$ such that $f\left(\partial V_{0}\right)=\partial V_{1}$ and $f\left(V_{0} \cap N\right) \subset V_{1}$; choose a compact set $K$ in $V_{0}$ and two points $a \in V_{0}$ and $b \in V_{1}$. Then, there exists a quasiregular mapping $f_{1}: V_{0} \rightarrow V_{1}$ such that 



Figure 4: In this case, intersection between $\partial f^{k}(U)$ and $\partial E$ never occurs, which is crucial for Lemma 3.1 to be applied in our case. We have drawn the cases $1<k<p$ (left) and $k=p$ (right). In both of them, the cycle $\widehat{\mathbb{C}} \backslash E, f(U), \ldots, f^{k}(U) \subset \widehat{\mathbb{C}} \backslash E$ appears in grey.

- $f_{1}=f$ in $V_{0} \cap N_{1}$, where $N_{1}$ is a neighbourhood of $\partial V_{0}$ with $N_{1} \subset N$;

- $f_{1}$ is holomorphic in a simply-connected neighbourhood of $K$;

- $f_{1}(a)=b$.

This Interpolation Lemma is a standard result and the details of its proof can be found in [5] or [10]. Applied to our case, it provides us with a quasiregular map $f_{1}: \widehat{\mathbb{C}} \backslash E \rightarrow f(U)$ which agrees with $f$ on $\partial E$, is holomorphic in a neighbourhood of $f^{k}(U)$ and satisfies $f_{1}(a)=b$.

Now we construct a map $f_{2}$ by setting $f_{2}=f$ on $E$ and $f_{2}=f_{1}$ on $\widehat{\mathbb{C}} \backslash E$, which makes it a quasi-regular map of $\widehat{\mathbb{C}}$, holomorphic in both a neighbourhood of $E$ and a neighbourhood of $\overline{f^{k}(U)}$, with a $k$-periodic point, given that $f_{2}^{k}(a)=f^{k-1}\left(f_{1}(a)\right)=f^{k-1}(b)=a$. Observe also that $f_{2}^{k}(\widehat{\mathbb{C}} \backslash$ $E)=f^{k}(U)$ and $\overline{f^{k}(U)} \subset \widehat{\mathbb{C}} \backslash E$; it follows that $f_{2}^{k}$ is a contraction and $a$ a global attractor for $f_{2}^{k}$ in $\widehat{\mathbb{C}} \backslash E$.

We may define an almost complex structure $\sigma$ by

$$
\sigma:= \begin{cases}\sigma_{0} & \text { on } f(U) \\ \left(f_{2}^{n}\right)^{*} \sigma_{0} & \text { on } f_{2}^{-n}(f(U)), \text { for } n \in \mathbb{N} \\ \sigma_{0} & \text { elsewhere }\end{cases}
$$

Observe that $\sigma=\sigma_{0}$ on $\bigcup_{i=1}^{k} f^{i}(U)$ (see Figure 5).

Furthermore, $\sigma$ is $f_{2}$-invariant by construction and has bounded distortion, since orbits pass through $\widehat{\mathbb{C}} \backslash\left(E \cup \overline{f^{k}(U)}\right)$ (the set where $f_{2}$ is not holomorphic) at most once. 


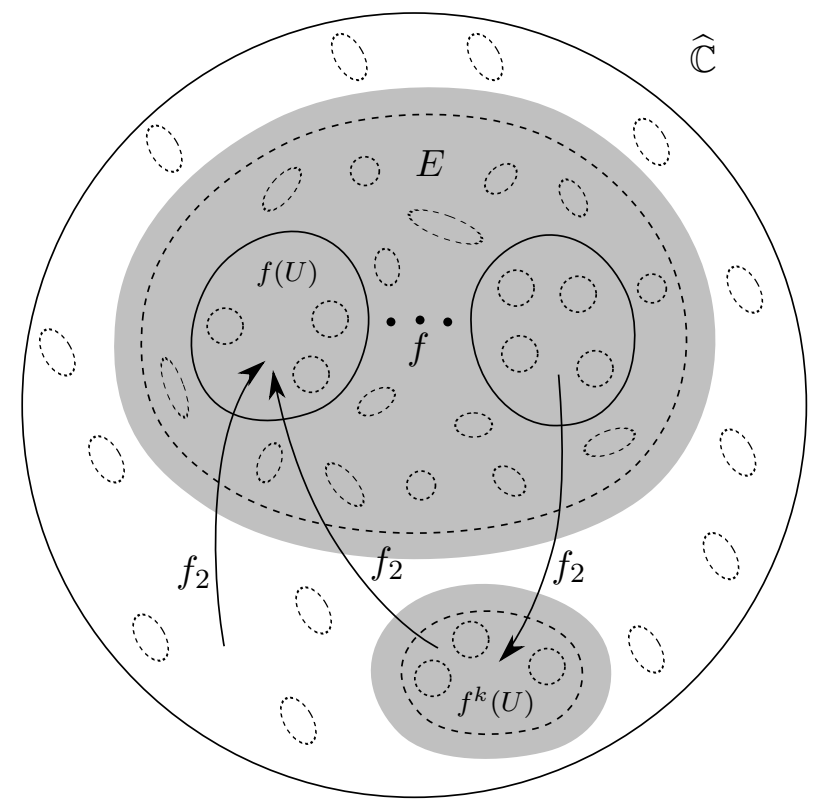

Figure 5: Construction of the almost complex structure $\sigma$. In grey we find the region where $f_{2}$ is holomorphic. Orbits pass through $\widehat{\mathbb{C}} \backslash\left(E \cup \frac{f^{k}(U)}{}\right)$ at most once.

REMARK. At this point, notice the importance of the fact that $f_{2}$ be defined to be holomorphic on a neighbourhood of $\overline{f^{k}(U)}$, which was only possible because $f^{k}(U)$ is a relatively compact subset of $\widehat{\mathbb{C}} \backslash E$.

These are precisely the hypothesis of Corollary 2.2, so there exists a map $g: \widehat{\mathbb{C}} \rightarrow \widehat{\mathbb{C}}$, holomorphic on the whole sphere - and hence rational-, which is conjugate to $f_{2}$ by some quasi-conformal homeomorphism $\phi$.

Now a theorem of Fatou ensures the existence of a weakly repelling fixed point $z_{0}$ of $g$, except when $\operatorname{deg} g=1$ and $g$ is an elliptic transformation. But $\phi(a)$ is an attracting $k$-periodic point of $g$, so this can never be the case.

Besides, the family $\mathcal{G}=\left\{\left.g^{n}\right|_{\phi(\widehat{\mathbb{C}} \backslash E)}\right\}_{n \geq 1}$ omits the open set $\phi(\widehat{\mathbb{C}} \backslash(E \cup$ $\left.\overline{f^{k}(U)}\right)$, therefore $\mathcal{G}$ is normal in $\phi(\widehat{\mathbb{C}} \backslash E)$ by Montel's Theorem, that is, $\phi(\widehat{\mathbb{C}} \backslash E) \subset \mathcal{F}(g)$. But weakly repelling fixed points belong to the Julia set, so $z_{0} \in \phi(E)$. Because such points are preserved under conjugacy, also $f_{2}$ has a weakly repelling fixed point $\phi^{-1}\left(z_{0}\right)$, in $E$; and so does $f$, since both functions coincide precisely on this set.

Case 2.2: $k=p$ and $\partial f^{p}(U) \cap \partial E \neq \emptyset$

For this case, let us first rename the elements of the periodic orbit and shift the sequence $\left\{U_{k}\right\}_{k}$ so that $\alpha \in \partial U \equiv \partial U_{n_{0}}$, i.e., so that $p \mid n_{0}$. More precisely, it is clear that there exists $0 \leq l<p$ such that $U \subset f^{l}(B)$; then, 
rename $B \equiv f^{l}(B), \alpha \equiv f^{l}(\alpha), U_{0} \equiv f^{l}\left(U_{0}\right)$ and define the sets $U_{1}, \ldots, U_{l-1}$ accordingly. Notice that $U_{0}, \ldots, U_{l}$ are all simply connected by construction. Since $p$ divides $n_{0}$, we can define $c:=n_{0} / p \in \mathbb{N}$, that is, the number of $f^{p}$ cycles from $U_{0}$ to $U_{n_{0}}$ (see Figure 6).

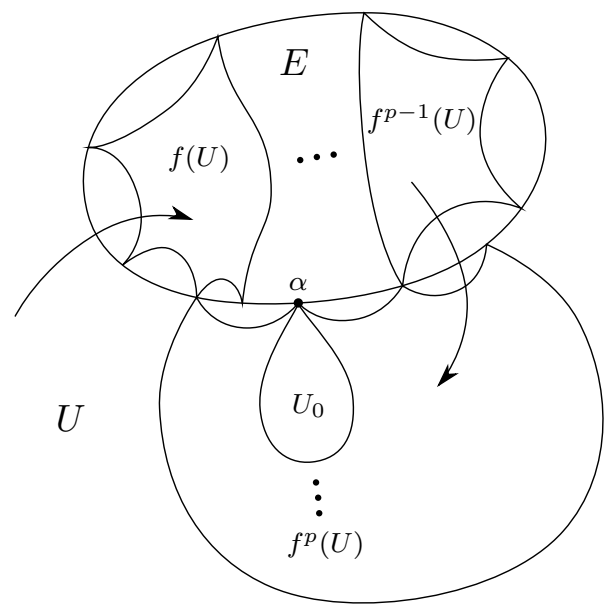

Figure 6: The shifted sequence $\left\{U_{k}\right\}_{k}$. From now on, this is the primary situation we should always bear in mind. The sets $U, f(U), \ldots, f^{p}(U)$ are the only ones in $\left\{U_{k}\right\}_{k}$ that will later play a role during the quasi-conformal surgery process. Their cyclic dynamics under the action of $f$ is also shown here.

Also, the sets $U_{k p+1}, \ldots, U_{(k+1) p-1} \subset E$ are necessarily bounded, so only those in the subsequence $U_{0}, U_{p}, U_{2 p}, \ldots$ might become unbounded from a certain one on. In particular, only the sets of the form $U_{k p+1}$ can have poles on their boundaries, and only the maps of the form $\left.f\right|_{U_{k p}}: U_{k p} \rightarrow U_{k p-1}$ can be of infinite degree.

Furthermore, notice that if some intersection $\partial U_{k_{1}} \cap \partial U_{k_{2}}$ contains a preimage of some pole, then the sets $U_{k_{1}}$ and $U_{k_{2}}$ necessarily belong to the same subsequence $U_{j} \subset U_{p+j} \subset U_{2 p+j} \subset \ldots$, that is, $k_{1} \equiv k_{2}(\bmod p)$ and either $U_{k_{1}} \subset U_{k_{2}}$ or $U_{k_{2}} \subset U_{k_{1}}$. In particular, only if this is the case can $\partial U_{k_{1}}$ and $\partial U_{k_{2}}$ share infinitely many preimages of $\alpha$. This will be a key point in later arguments.

We have seen that the fact that $\partial f^{p}(U)$ and $\partial E$ did not share any contact point was crucial for the quasi-conformal surgery construction of Case 2.1 (see Remark 3). Now, the condition $\partial f^{p}(U) \cap \partial E \neq \emptyset$ is exactly given by hypothesis, so some extra work must be done, in the sense of modifying slightly some sets, in order to start the surgical process proper.

On the other hand, notice that the sets $\left\{U_{k}\right\}_{k}$ are in some sense arbitrary, since they were constructed by repeatedly pulling-back $U_{0}$, chosen arbitrarily. Also, notice that once these sets (and $E$ ) have been defined and during the process of quasi-conformal surgery (that is to say, from the construction 
of the auxiliar quasi-regular maps on), the only sets in this sequence with a role to play are $U, f(U), \ldots, f^{k}(U)$ (or rather $U, f(U), \ldots, f^{p}(U)$ for the current case).

Thus, it seems that we can modify these sets $U, f(U), \ldots, f^{p}(U)$ slightly and only close to the odd contact points, so that their boundaries share as little points as possible - the following result provides us with such modification. Its proof is rather technical and will be given separately, at the end of this section.

Proposition 3.2. In the situation described hitherto, there exists a connected multiply-connected set $\mathcal{U} \subset U$ such that $f^{p}(\mathcal{U})$ is simply connected, $\overline{f^{p}(\mathcal{U})} \subset \mathcal{U} \cup\{\alpha\}$ and $\partial f^{p}(\mathcal{U}) \cap \mathcal{J}(f)=\{\alpha\}$.

Now call $\mathcal{E}$ the bounded component of $\widehat{\mathbb{C}} \backslash \mathcal{U}$ that contains $E$. The point $\alpha$ need not be on $\partial \mathcal{E}$, so it could happen that $\partial f^{p}(\mathcal{U}) \cap \partial \mathcal{E}=\emptyset$. Were that the case, notice that $\overline{f^{p}(\mathcal{U})} \subset \widehat{\mathbb{C}} \backslash \mathcal{E}$ and therefore we could just repeat the surgery process of Case 2.1 -replacing $U$ and $E$ by their respective modifications - to find a weakly repelling fixed point of $f$.

Otherwise, we have $\partial f^{p}(\mathcal{U}) \cap \partial \mathcal{E}=\{\alpha\}$ and, as there seems to be no neat way to separate $\mathcal{E}$ from $\alpha$, we will just work with a small extension of $\mathcal{E}$ whose interior contains $\alpha$. More precisely, we first define $V_{0}:=\widehat{\mathbb{C}} \backslash \mathcal{E}$ and $V_{1}:=f(\mathcal{U}) \subset E \subset \mathcal{E}$, and use the Interpolation Lemma 3.1 to find a quasi-regular map $f_{1}: \widehat{\mathbb{C}} \backslash \mathcal{E} \rightarrow f(\mathcal{U})$, as usual-however, notice that we marked no compact set $K$ nor points $a$ and $b$, since now $f_{1}$ need not be holomorphic in any subset of $\widehat{\mathbb{C}} \backslash \mathcal{E}$. Also, recall that $f_{1}$ actually agrees with $f$ in a neighbourhood $N_{1}$ of $\partial \mathcal{E}$-call $\mathcal{N}:=\mathcal{E} \cup N_{1}$, a neighbourhood of $\mathcal{E}$.

Lemma 3.3. There exist a sufficiently small neighbourhood of $\alpha$ in $f^{p}(\mathcal{U})$, $\mathcal{W}^{*}$, an open neighbourhood of $\mathcal{E} \cup \overline{f^{p}\left(\mathcal{W}^{*}\right)}$ in $\mathcal{N}, \mathcal{E}^{*}$, and a quasi-conformal map $h: \widehat{\mathbb{C}} \rightarrow \widehat{\mathbb{C}}$ such that

- $\overline{\mathcal{W}^{*}} \subset \mathcal{N}$;

- $\overline{f^{p}\left(\mathcal{W}^{*}\right)} \subset \mathcal{E}^{*}$ and $\overline{\mathcal{E}^{*} \cap \mathcal{W}^{*}} \backslash \partial f^{p}(\mathcal{U}) \subset \mathcal{W}^{*}$;

- $h=$ id in $\mathcal{E}^{*}$ and $h\left(f^{p}(\mathcal{U})\right) \subset \mathcal{W}^{*}$.

Roughly speaking, the map $h$ pushes the points in $f^{p}(\mathcal{U})$ towards $\mathcal{E}$, but will leave points there untouched so that the action of any post-composed map be preserved entirely (see Figure 7).

Proof. We define the set $\mathcal{W}^{*}$ as the connected component of $f^{-(c-1) p}\left(W_{R}\right)$ in $f^{p}(\mathcal{U})$ that has $\alpha$ on the boundary, with $R$ so large as for $\overline{\mathcal{W}^{*}} \subset \mathcal{N}$ (see the construction of $\mathcal{W}$ in Subsection 3.1). By construction, it is a neighbourhood of $\alpha$ in $f^{p}(\mathcal{U})$, i.e., $\alpha \notin \overline{f^{p}(\mathcal{U}) \backslash \mathcal{W}^{*}}$, and $f^{p}\left(\overline{\mathcal{W}^{*}}\right) \subset \mathcal{W}^{*} \cup\{\alpha\}$. In particular, the existence of one such $\mathcal{E}^{*}$ follows from the latter. 




Figure 7: The case where $\partial \mathcal{V} \cap \partial \mathcal{E}=\{\alpha\}$, with the sets $\mathcal{N}, \mathcal{W}^{*}$ (light-shaded), $f^{p}\left(\mathcal{W}^{*}\right)$ (dark-shaded) and $\mathcal{E}^{*}$. Notice that points in $f^{p}\left(\mathcal{W}^{*}\right)$ will never leave $\mathcal{E}^{*}$ under the action of $f$.

Now let $S$ be the simply-connected open set $f^{p}(\mathcal{U}) \backslash \overline{\mathcal{E}^{*}}$ with a marked boundary segment at $l:=\partial S \cap \partial \mathcal{E}^{*}$. There exists a (conformal) Riemann map $\varphi: S \rightarrow Q$ that sends $l$ to one of the sides of the open unit square $Q$. Consider a (quasi-conformal) homothetic transformation $\tilde{h}_{0}: \bar{Q} \rightarrow \tilde{h}_{0}(\bar{Q})$ such that $\left.\tilde{h}_{0}\right|_{\varphi(l)}=\mathrm{id}$ and $\tilde{h}_{0}(Q) \cap \varphi\left(S \cap \partial \mathcal{W}^{*}\right)=\emptyset$.

Finally, define the conjugate map $h_{0}:=\varphi^{-1} \circ \tilde{h}_{0} \circ \varphi: S \rightarrow h_{0}(S)$, which is quasi-conformal (see Figure 8). Notice that $\left.h_{0}\right|_{l}=\mathrm{id}$, so we can define

$$
h:= \begin{cases}h_{0} & \text { on } S=f^{p}(\mathcal{U}) \backslash \overline{\mathcal{E}^{*}} \\ \text { id } & \text { on } \overline{\mathcal{E}^{*}}\end{cases}
$$

and extend it quasi-conformally to a map $h: \widehat{\mathbb{C}} \rightarrow \widehat{\mathbb{C}}$.

Now consider the quasi-regular map $f_{2}: \widehat{\mathbb{C}} \rightarrow \widehat{\mathbb{C}}$ defined as

$$
f_{2}:=\left\{\begin{array}{ll}
f \circ h & \text { on } \mathcal{E}^{*} \\
f_{1} \circ h & \text { on } \widehat{\mathbb{C}} \backslash \mathcal{E}^{*}
\end{array}= \begin{cases}f & \text { on } \mathcal{E}^{*} \\
f_{1} \circ h & \text { on } \widehat{\mathbb{C}} \backslash \mathcal{E}^{*}\end{cases}\right.
$$

Also, consider the (shrinking) $f_{2}$-cycle $C:=f\left(\mathcal{W}^{*}\right) \cup \ldots \cup f^{p}\left(\mathcal{W}^{*}\right) \subset \mathcal{E}^{*}$. Indeed, it is cyclic because $f_{2}(C)=f(C) \subset C$ (see Figure 9). 

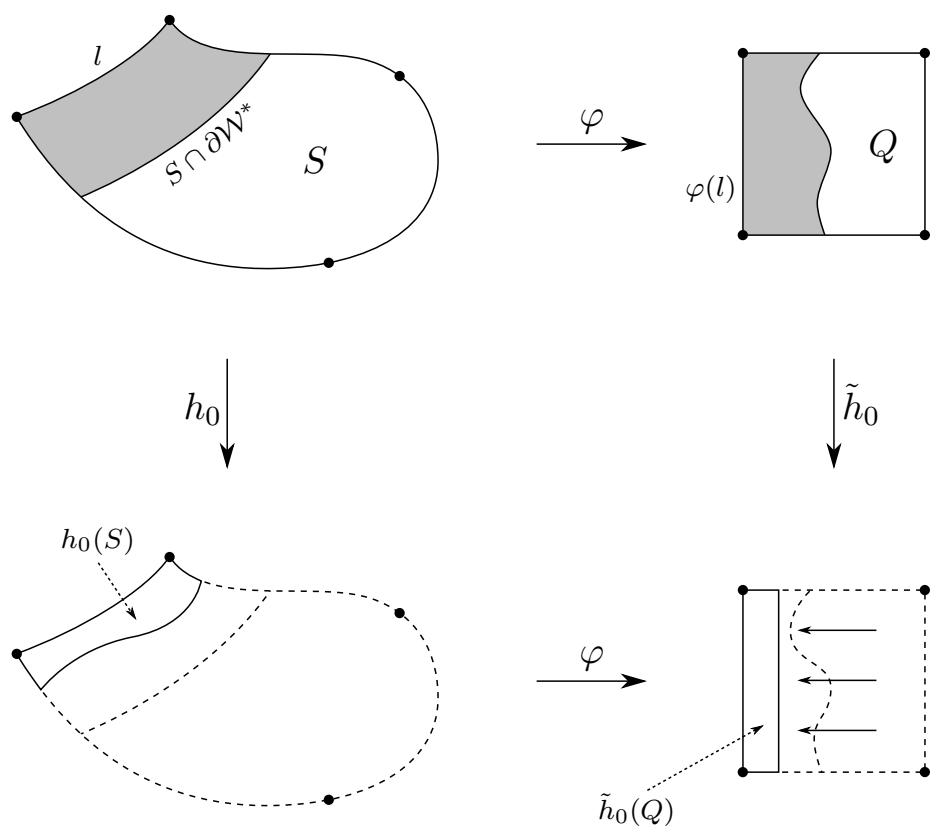

Figure 8: For the construction of the map $h: \widehat{\mathbb{C}} \rightarrow \widehat{\mathbb{C}}$, we first define an auxiliary map $h_{0}: S \rightarrow h_{0}(S)$ as a conjugation of a quasi-conformal map on $Q$, where it is easy to define the desired local dynamics. In grey we find $S \cap \mathcal{W}^{*}$ (and its $\varphi$-image), the subset where we want $h_{0}(S)$ to end up.

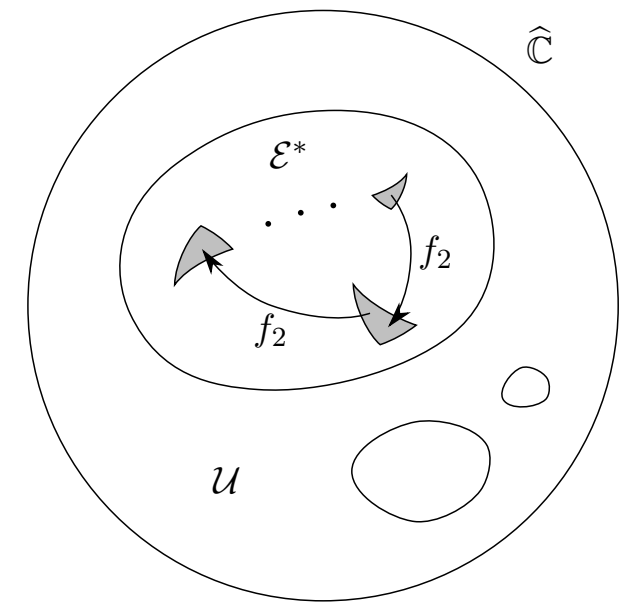

Figure 9: The action of $f_{2}$ on the cycle $C$, shaded. Notice that $f^{p}\left(\mathcal{W}^{*}\right) \subset \mathcal{W}^{*}$, so its $f_{2}$-image falls again in $f\left(\mathcal{W}^{*}\right)$.

Setting $X:=\widehat{\mathbb{C}} \backslash \mathcal{E}^{*}$, orbits of $f_{2}$ pass through $X$ at most twice, since

$$
\begin{aligned}
\ldots & \stackrel{f_{2}}{\longmapsto} f_{2}^{-1}(X) \stackrel{f_{2}}{\longmapsto} X \stackrel{h}{\longmapsto} X \subset \widehat{\mathbb{C}} \backslash \mathcal{E} \stackrel{f_{1}}{\longmapsto} f(\mathcal{U}) \stackrel{f_{2}^{p-1}}{\longmapsto} f^{p}(\mathcal{U}) \\
& \stackrel{h}{\longmapsto} \mathcal{W}^{*} \stackrel{f}{\longmapsto} f\left(\mathcal{W}^{*}\right) \subset C \stackrel{f_{2}}{\longmapsto} C \stackrel{f_{2}}{\longmapsto} \cdots \subset \widehat{\mathbb{C}} \backslash X .
\end{aligned}
$$


Define the almost complex structure

$$
\sigma:= \begin{cases}\sigma_{0} & \text { on } C \\ \left(f_{2}^{n}\right)^{*} \sigma & \text { on } f_{2}^{-n}\left(f\left(\mathcal{W}^{*}\right)\right), n \in \mathbb{N} \\ \sigma_{0} & \text { elsewhere }\end{cases}
$$

which clearly is $f_{2}$-invariant by definition, and has bounded dilatation since $f_{2}$ fails to be holomorphic only at most twice. Therefore we can use Corollary 2.2 to find a rational map $g: \widehat{\mathbb{C}} \rightarrow \widehat{\mathbb{C}}$ conjugate to $f_{2}$ by a quasi-conformal homeomorphism $\phi$.

Thus,

$$
\overline{g^{p+1}(\phi(X))}=\overline{\phi\left(f_{2}\left(f_{2}^{p}(X)\right)\right)} \subset \overline{\phi\left(f_{2}\left(f^{p}(\mathcal{U})\right)\right)} \subset \overline{\phi(C)} \subset \phi(C) \cup\langle\phi(\alpha)\rangle,
$$

so $\phi(X)$ is contained in the basin of an attracting or parabolic point. By Fatou's theorem, $g$ has a weakly repelling fixed point in $\phi(\widehat{\mathbb{C}} \backslash X)=\phi\left(\mathcal{E}^{*}\right)$, hence $f$ has a weakly repelling fixed point in $\mathcal{E}^{*}$.

\subsection{Proof of Proposition 3.2}

When removing points of $\partial f^{p}(U) \cap \partial E$, there is a particular point we cannot ignore - that is $\alpha$ itself: Because its attracting dynamics in a whole petal contained in the parabolic basin (Fatou coordinates about a parabolic point), if we redefined $U$ as some $\widetilde{U}$ in such a way that $\alpha$ were not on its boundary, then points close to $\alpha$ would become even closer under the action of $f^{p}$, and the condition $f^{p}(\widetilde{U}) \subset \widetilde{U}$ would be lost (see Figure 10).

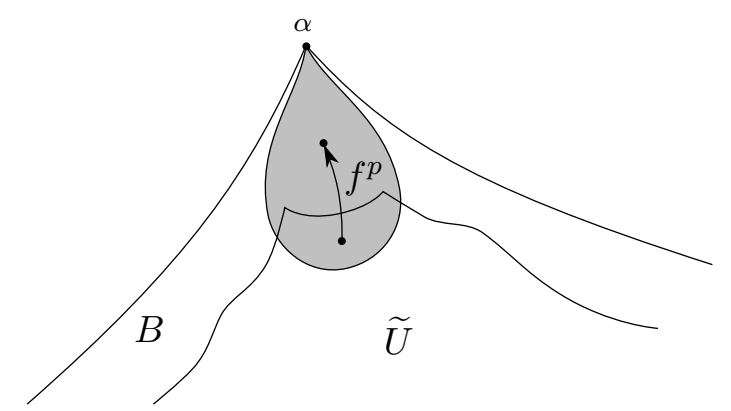

Figure 10: If the new set $\widetilde{U}$ left out some neighbourhood of $\alpha$, there would be points in it stepping outside it under $f^{p}$. The shaded set represents the attracting petal attached to $\alpha$ given by the Fatou coordinates.

Rather, for the construction of one such $\mathcal{U}$ we need to modify the sets $U, f(U), \ldots, f^{p}(U)$ close to the contact points between their boundaries except those in the cycle $\langle\alpha\rangle$ (see Figure 11). 

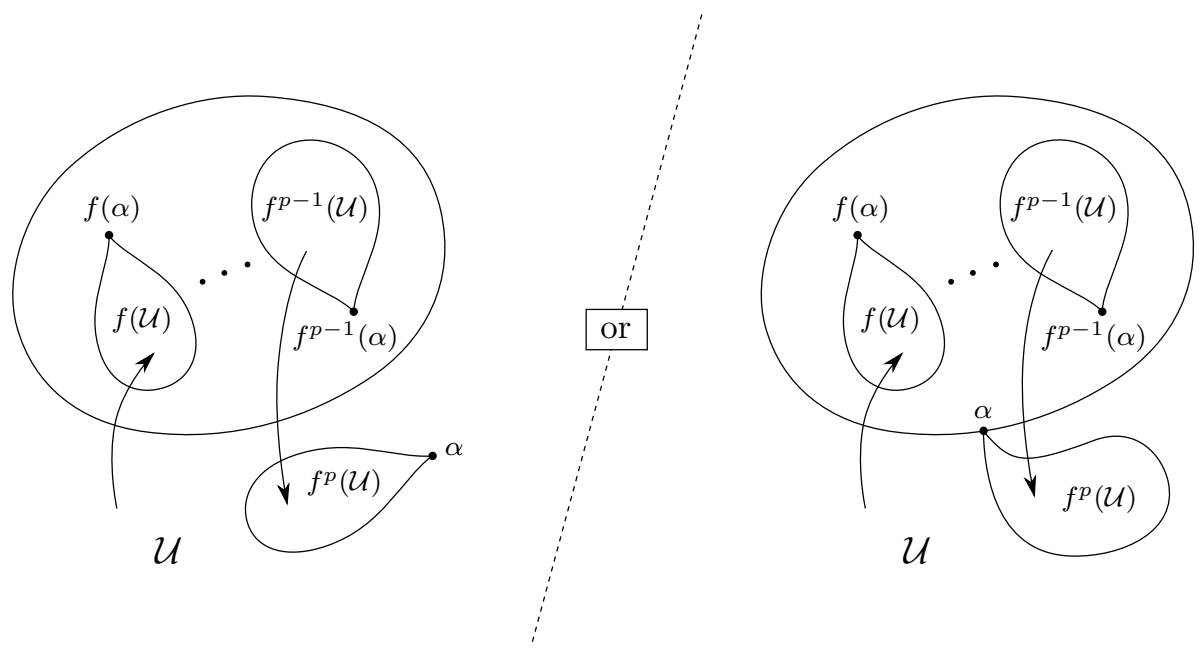

Figure 11: The situation we want, with the points in $\langle\alpha\rangle$ marked. Notice that a priori we do not know whether $\alpha$ is on $\partial E$ or not, since the set $E$ was chosen arbitrarily as one of the bounded connected components of the complement of $U$; in particular, $\alpha$ could even happen to be on the boundary of the unbounded component of the complement of $\mathcal{U}$. It is clear that surgery cannot be used just as in Case 2.1 when $\partial f^{p}(\mathcal{U}) \cap \partial E$ remains nonempty (right-hand side figure).

When doing so, it is clear that if the point $\alpha$ lies not on the intersection $\partial f^{p}(\mathcal{U}) \cap \partial E$ (Figure 11, left), the situation is then identical to that of Case 2.1 , and therefore we can conclude the case following an analogous surgical procedure. In case $\alpha$ does belong to $\partial f^{p}(\mathcal{U}) \cap \partial E$ (Figure 11, right), yet one last auxiliar map shall be defined in order to finish the proof with a different quasi-conformal surgery argument.

Let us now construct the modification of $U, f(U), \ldots, f^{p}(U)$. The idea is the following: Since the ultimate aim of such modification is to eliminate contact points between $\partial f^{p}(U)$ and $\partial E$, it suffices to modify only the set $U_{n_{0}-p} \equiv f^{p}(U)$ and redefine the sets $U_{n_{0}-p+1} \equiv f^{p-1}(U), \ldots, U_{n_{0}} \equiv U$ by repeatedly pulling-back this first modification, appropriately. Of course if the changes on these sets are arbitrarily small, and, therefore, the new sets are arbitrarily close to the original ones, their respective connectivities are also to be preserved (see Figure 12).

Following such reasoning, one could think that the modification of $f^{p}(U)$, which we can call $\mathcal{V}$, could simply be obtained by removing from $f^{p}(U)$ a disc of arbitrarily small radius centered at every contact point between $\partial f^{p}(U)$ and $\partial E$ (see Figure 13).

But of course we want to keep the property $f^{p}(U) \subset U$ for the subsequent surgical work, and, if we just removed those discs taking no control whatsoever over their preimages, such inclusion could be lost: Consider a 


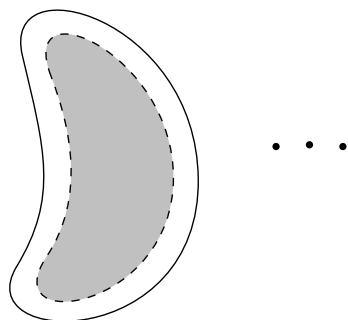

$f^{p}(U)$

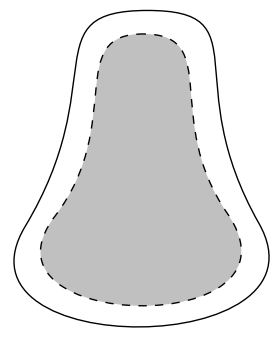

$f(U)$

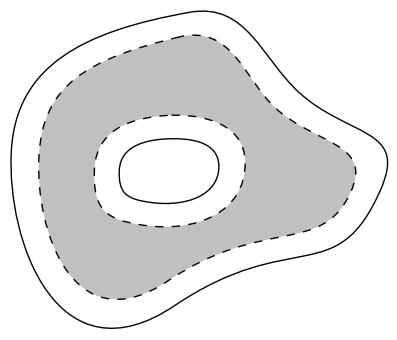

$U$

Figure 12: The set $U$ is multiply connected and so is its modification (shaded here) if it differs not much from $U$. Similarly, the sets $f(U), \ldots, f^{p}(U)$ are simply connected and so are their modifications.



Figure 13: The shaded set represents a first attempt towards the construction of $\mathcal{V}$.

point $a \in \mathcal{A}:=\partial f^{p}(U) \cap \partial E \backslash\{\alpha\} \subset \mathcal{J}(f)$ - for instance some $a \in \mathcal{O}^{-}(\alpha)$ with some preimage $b \in f^{-p}(a)$ on the same set $\mathcal{A}$. If, when defining $\mathcal{V}$, and since $a, b \in \mathcal{A}$, we removed from $f^{p}(U)$ discs of small radius centered at these points, say $B_{\varepsilon}(a)$ and $B_{\varepsilon}(b)$, and if the preimage of $B_{\varepsilon}(a)$ under $f^{p}$ happened to become so big as to contain points in the complement of $B_{\varepsilon}(b)$, then there would be points $z_{0} \in\left(f^{-p}\left(B_{\varepsilon}(a)\right) \cap f^{p}(U)\right) \backslash B_{\varepsilon}(b)$ such that $f^{p}\left(z_{0}\right) \in B_{\varepsilon}(a) \subset \widehat{\mathbb{C}} \backslash \mathcal{V}$, that is to say, $z_{0} \notin f^{-p}(\mathcal{V})$ and, therefore, we would have $z_{0} \in \mathcal{V} \backslash f^{-p}(\mathcal{V}) \neq \emptyset$, which is precisely what we want to avoid. (See Figure 14.)

This very description of the problem with the preimages of points we 


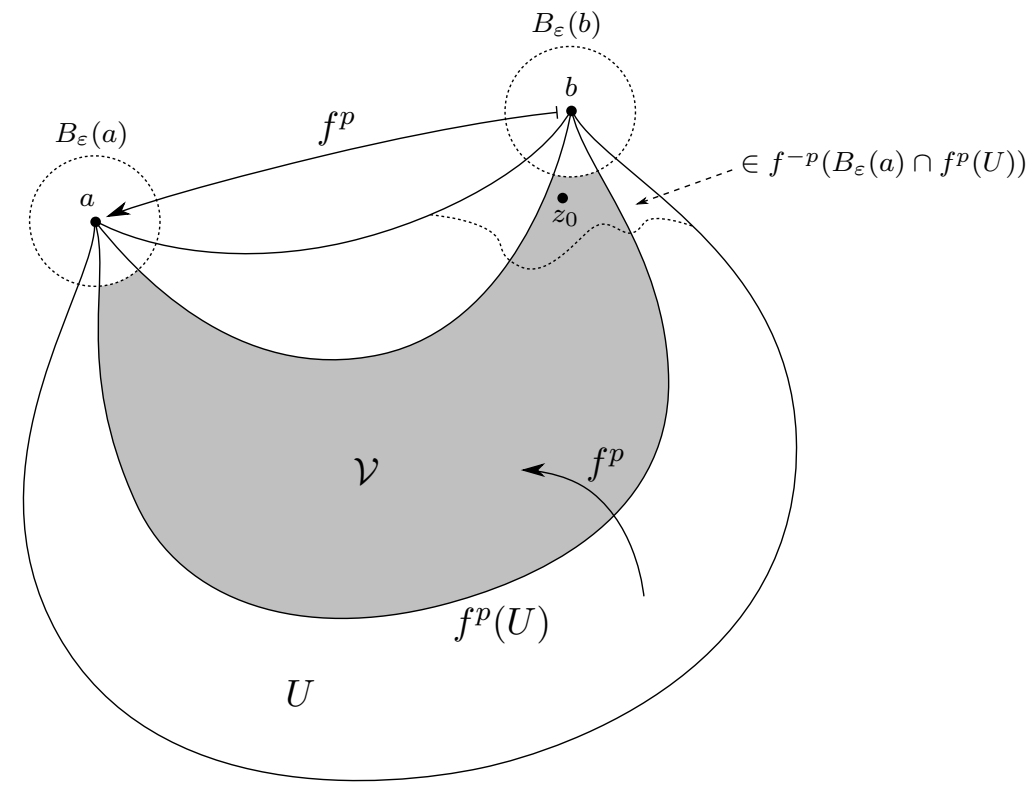

Figure 14: We want to keep $f^{p}(U) \subset U$ after the modification, i.e., we want a set $\mathcal{V}$ such that $\mathcal{V} \subset f^{-p}(\mathcal{V})$. However, if we defined it as the shaded set in this figure, there would exist points $z_{0} \in \mathcal{V} \backslash f^{-p}(\mathcal{V})$ - so we need to take some control over the preimages of the discs we remove from $f^{p}(U)$.

remove from $f^{p}(U)$ for the construction of $\mathcal{V}$ also provides us with a hint about how to solve it, since, in the previous example, it would have been enough to take $f^{-p}\left(B_{\varepsilon}(a)\right) \cap f^{p}(U)$ instead of $B_{\varepsilon}(b)$ so as to avoid points like $z_{0}$.

In other words, we must also exclude from $\mathcal{V}$ all the points in $f^{p}(U)$ whose $f^{p}$-image falls on points we "already" removed from $f^{p}(U)$. In fact, this generates, in turn, more points whose preimage need be controlled; and so on. Regardless of what may be expected, this is not an endless recurrent process, since $f^{p}(U) \equiv U_{n_{0}-p} \subset f^{-n_{0}+p}\left(U_{0}\right)$ and, therefore, after $n_{0}-p$ iterations all the points in $f^{p}(U)$ happen to be close to $\alpha$ - precisely in $U_{0}$, and we will see that we can make $U_{0} \subset \mathcal{V}$ provided that the radius of the discs initially removed from $f^{p}(U)$ is small enough (see Figure 15). At the same time, we shall be careful when taking all these preimages, since they could become so big as to impede the construction of $\mathcal{V}$.

For all $\varepsilon>0$, let

$$
V_{\varepsilon}:=f^{p}(U) \backslash \bigcup_{a \in \mathcal{A}} \bigcup_{k=0}^{c-2} f^{-k p}\left(B_{2^{k} \varepsilon}(a)\right) .
$$

The remaining part of the proof consists on showing that, for $\varepsilon$ small enough, the set $\mathcal{V} \equiv V_{\varepsilon}$ is exactly the one we want. 




Figure 15: Given a sequence of points $a_{l} \mapsto \cdots \mapsto a_{2} \mapsto a_{1}$ on $\mathcal{A}$, with $1 \leq l<c$ (since $f^{p}(U) \equiv U_{n_{0}-p}=U_{(c-1) p}$ and $f^{p}\left(\overline{U_{0}}\right) \subset U_{0} \cup\{\alpha\}$, so $\partial f^{p}(U)$ cannot contain preimages of higher order), the points $z_{0} \in f^{-(l-1) p}\left(B_{\varepsilon}\left(a_{1}\right)\right)$ will eventually fall inside $U_{0} \subset \mathcal{V}$, therefore we need not worry about their preimages any more.

First of all notice that $f^{p}\left(V_{\varepsilon}\right) \subset V_{\varepsilon}$ by definition. Now we will show that the preimages $f^{-k p}\left(B_{2^{k} \varepsilon}(a)\right)$ can be controlled in such a way that none of them reaches the point $\alpha$, otherwise excluded from $V_{\varepsilon}$. The following lemma gives sufficient conditions for this not to happen.

Lemma 3.4. In the situation described hitherto, there exists $\varepsilon_{0}>0$ such that $\alpha \in \partial V_{\varepsilon}$ for all $\varepsilon<\varepsilon_{0}$.

Before its proof, we define two sets which, because of their importance, will be used also beyond this result. These sets are both neighbourhoods (in $f^{p}(U)$ ) of $\alpha$ and provide useful information about the dynamics of $f^{p}$ close to this point.

The first set to be constructed, $\mathcal{C}$, is a neighbourhood of $\alpha$ whose boundary is clean of points of $\mathcal{A}$. For this, notice that $\mathcal{A}$ consists only of points of $\mathcal{O}^{-}(\alpha)$ and $\mathcal{O}^{-}(\infty)$, since $\mathcal{A} \subset \partial f^{p}(U) \cap \mathcal{J}(f)$ and, by construction of the sequence $\left\{U_{k}\right\}_{k}$, we have $f^{(c-1) p}\left(\partial f^{p}(U)\right)=\partial U_{0} \subset \mathcal{F}(f) \cup\{\alpha\}$. More precisely,

$$
\mathcal{A} \subset \bigcup_{1 \leq k<c}\left(f^{-k p}(\alpha) \cup f^{-(k-1) p}(\infty)\right)
$$

or, simply,

$$
\mathcal{A} \subset f^{-(c-1) p}(\alpha) \cup \bigcup_{k=0}^{c-2} f^{-k p}(\infty)
$$


if we take into account that $\alpha$ is $q$-periodic and so $p$-periodic. In particular, the set $\mathcal{A}$ finds its accumulation points only in $\bigcup_{k=0}^{c-2} f^{-k p}(\infty)$, and the points in $f^{-(c-1) p}(\alpha) \cap \mathcal{A}$ are all isolated in $\mathcal{A}$ (since $f^{-(c-1) p}(\alpha) \cap \bigcup_{k=0}^{c-2} f^{-k p}(\infty)=\emptyset$ because $\alpha$ is a periodic point). In the same way, since $\alpha$ is not an accumulation point of $\mathcal{A}$, there exists a simply-connected open sector $\mathcal{C} \subset f^{p}(U)$ such that $\alpha \in \partial \mathcal{C}, \alpha \notin \overline{f^{p}(U) \backslash \mathcal{C}}$ and $\overline{\mathcal{C}} \cap \mathcal{A}=\emptyset$ (see Figure 16). Actually, we can still shrink it slightly so that $\mathcal{A}$ does not meet a whole (sufficiently small) neighbourhood of $\overline{\mathcal{C}}$ - we will use this later, in order to see some technical detail.

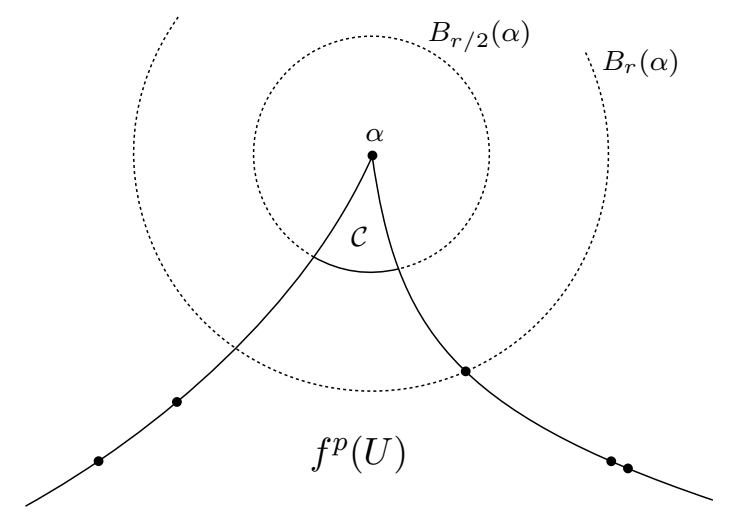

Figure 16: The non-labelled points represent the set $\mathcal{A}$. Since they never accumulate on $\alpha$, there certainly exists such an open set $\mathcal{C}$, as shown. Furthermore, because $\alpha$ is a parabolic point, in a sufficiently small neighbourhood of it $f^{p}(U)$ is essentially a wedge like that of an attracting petal, so we can even take $\mathcal{C}$ as $B_{r}(\alpha) \cap f^{p}(U)$ with $r$ so small as for $\mathcal{C}$ to be connected and $\overline{\mathcal{C}} \cap \mathcal{A}=\emptyset$. Even more, taking $\mathcal{C}=B_{r / 2}(\alpha) \cap f^{p}(U)$ we ensure not only its closure but also a whole neighbourhood of $\overline{\mathcal{C}}$ free from points of $\mathcal{A}$.

On the other hand, we want to construct another neighbourhood of $\alpha$ in $f^{p}(U)$, to be called $\mathcal{W}$, with dynamics similar to that of $U_{0}$ in the sense that $f^{p}(\overline{\mathcal{W}}) \subset \mathcal{W} \cup\{\alpha\}$; in other words, the set $\mathcal{W}$ will control those points in $f^{p}(U)$ that happen to be already close to the point $\alpha$. Notice that we cannot take $U_{0}$ itself as $\mathcal{W}$ because $U_{0}$ need not be a neighbourhood of $\alpha$ in $f^{p}(U)$, that is, $\alpha \in \overline{f^{p}(U) \backslash U_{0}}$ in general; but the construction of $U_{0}$ does inspire the use of Fatou coordinates in order to provide $\mathcal{W}$ with its same dynamics. More precisely, we will construct a subset of $U_{0}$ in a very similar fashion and then define $\mathcal{W}$ as an appropriate preimage of it in $f^{p}(U)$.

In fact, for all $R>L$, let

$$
W_{R}:=H^{-1}(\{w \in \mathbb{C}: \operatorname{Re} w>L, \operatorname{Re} w+|\operatorname{Im} w|>R\}) \subset U_{0},
$$

where recall that $H(z)=-1 / \nu a z^{\nu}$ conjugates the maps $f^{p}$ and $T(w)=$ $w+1+O\left(w^{-1 / \nu}\right)$, and $L>0$ is large enough for $f^{p}$ to be injective on $U_{0}$ 
(see Figure 17).
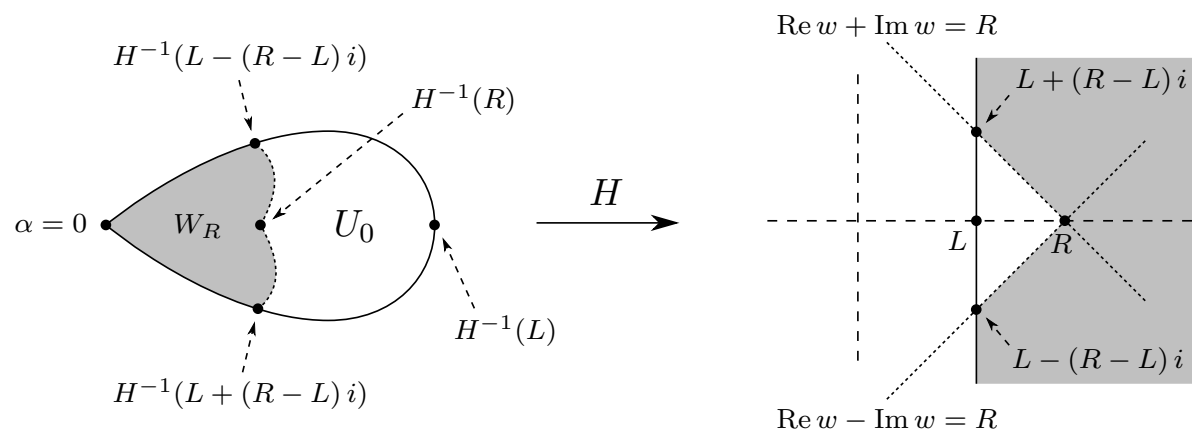

Figure 17: Using the same Fatou coordinates setting as in the construction of $U_{0}$, we can define $W_{R}$ as a subset of it in such a way that $f^{p}$ keeps its injectivity also in the subset. By taking $R$ sufficiently large, $W_{R}$ can be embedded in any (arbitrarily small) neighbourhood of $\alpha$.

It is clear that since we took $L$ so large as for $T(w) \approx w+1$ and $f^{p}\left(\overline{U_{0}}\right) \subset$ $U_{0} \cup\{\alpha\}$, then, for any $R>L$, also $f^{p}\left(\overline{W_{R}}\right) \subset W_{R} \cup\{\alpha\}$ holds. Moreover, $W_{R}$ is a neighbourhood of $\alpha$ in $U_{0}$ (i.e., $\alpha \notin \overline{U_{0} \backslash W_{R}}$ ), since $H(\alpha)=\infty$ and $H\left(\overline{U_{0} \backslash W_{R}}\right)=\{w \in \mathbb{C}: \operatorname{Re} w \geq L, \operatorname{Re} w+|\operatorname{Im} w| \leq R\}$, which is a compact set.

Consider now the connected component of the preimage $f^{-(c-1) p}\left(W_{R}\right)$ in $f^{p}(U)$ that has $\alpha$ on the boundary (or, equivalently, contains $W_{R}$ ). If $R$ were close to $L$, then $W_{R}$ would be close to $U_{0}$ and its preimage close to $f^{p}(U)$, so the character of neighbourhood of $\alpha$ would be lost. Let us show, then, that we can choose a sufficiently large $R$ in such a way that this preimage lies even inside the just-constructed neighbourhood $\mathcal{C}$ : Consider the image set $f^{(c-1) p}(\mathcal{C}) \subset U_{0}$; notice that $\alpha \notin \overline{U_{0} \backslash f^{(c-1) p}(\mathcal{C})}$ since, by construction of $\mathcal{C}$, there are no preimages of $\alpha$ on $\overline{\mathcal{C}}$. Therefore, there exists $R_{0}>L$ such that $W_{R} \subset f^{(c-1) p}(\mathcal{C}) \subset U_{0}$ for any $R>R_{0}$ (see Figure 18). Define $\mathcal{W}$ as the connected component of $f^{-(c-1) p}\left(W_{R}\right)$ in $f^{p}(U)$ that has $\alpha$ on the boundary, for $R>R_{0}$, and thus $\mathcal{W} \subset \mathcal{C}$. It follows that $f^{p}(\overline{\mathcal{W}}) \subset \mathcal{W} \cup\{\alpha\}$ and $\alpha \notin \overline{f^{p}(U) \backslash \mathcal{W}}$, since, once again, $\overline{\mathcal{C}} \cap \mathcal{A}=\emptyset$.

This concludes the construction of the sets $\mathcal{C}$ and $\mathcal{W}$, so we are now in a position to prove Lemma 3.4.

Proof of Lemma 3.4. Consider one of the preimages $f^{-k p}\left(B_{2^{k}}(a)\right)$ and suppose that $\alpha \in f^{-k p}\left(B_{2^{k} \varepsilon}(a)\right)$. If this were the case, and because $\alpha \in \partial \mathcal{W}$, we would have that $f^{-k p}\left(B_{2^{k} \varepsilon}(a)\right) \cap \mathcal{W} \neq \emptyset$; so let $z_{0} \in f^{-k p}\left(B_{2^{k}}(a)\right) \cap \mathcal{W}$. Then,

$$
\begin{array}{cccc}
\mathcal{C} \supset \mathcal{W} & \supset f^{p}(\mathcal{W}) & \supset \cdots & f^{k p}(\mathcal{W}) \\
\mathcal{U} & \Psi & & \mathcal{U} \\
z_{0} & f^{p}\left(z_{0}\right) & & f^{k p}\left(z_{0}\right)
\end{array}
$$




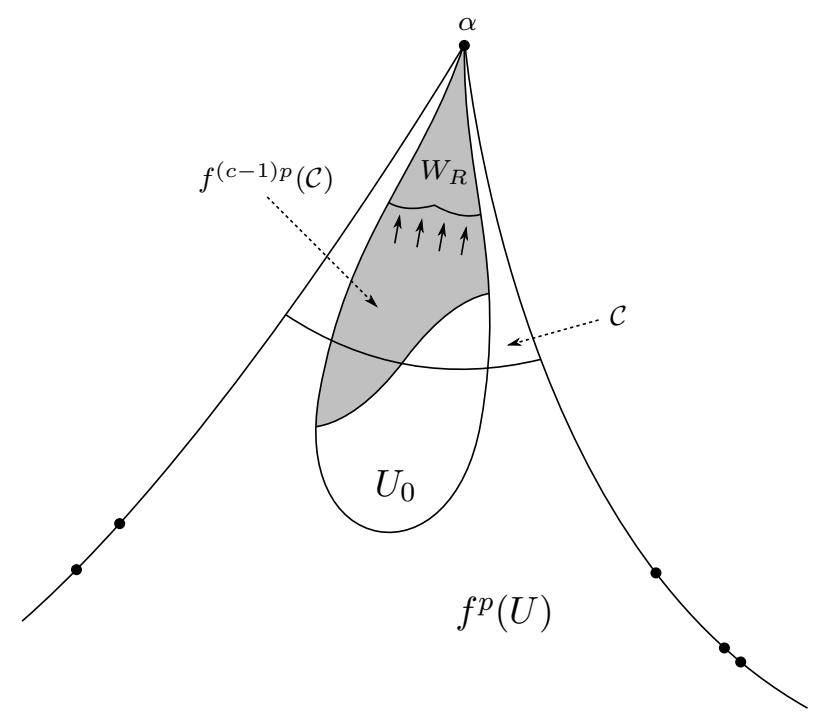

Figure 18: Since $\alpha \notin \overline{U_{0} \backslash f^{(c-1) p(\mathcal{C})}}$, the set $W_{R}$ can be shrunk arbitrarily until $W_{R} \subset f^{(c-1) p}(\mathcal{C})$. Notice that $f^{(c-1) p}(\mathcal{C})$, shaded here, need not be contained in $\mathcal{C}$, so $\mathcal{C}$ itself or even an image of it serve not as $W_{R}$.

that is to say, $f^{k p}\left(z_{0}\right) \in \mathcal{C}$. On the other hand, from the fact that $z_{0} \in$ $f^{-k p}\left(B_{2^{k} \varepsilon}(a)\right) \cap \mathcal{W}$ it also follows that $f^{k p}\left(z_{0}\right) \in B_{2^{k} \varepsilon}(a)$; therefore, the point $f^{k p}\left(z_{0}\right)$ would belong to both sets: $f^{k p}\left(z_{0}\right) \in \mathcal{C} \cap B_{2^{k}}(a)$.

However, since $\mathcal{A}$ does not meet some neighbourhood of $\overline{\mathcal{C}}$, it is clear that there exists $\varepsilon_{0}>0$ such that $\mathcal{C} \cap B_{2^{k}}(a)=\emptyset$ for any $\varepsilon<\varepsilon_{0}$ and $a \in \mathcal{A}$. Therefore, it suffices to take $\varepsilon<\varepsilon_{0}$ to obtain $f^{k p}\left(z_{0}\right) \notin \mathcal{C} \cap B_{2^{k} \varepsilon}(a)=\emptyset$ and $\alpha \notin f^{-k p}\left(B_{2^{k} \varepsilon}(a)\right)$. But $\alpha$ does belong to $\partial f^{p}(U)$ so, right from the definition of $V_{\varepsilon}$, we have $\alpha \in \partial V_{\varepsilon}$ for all $\varepsilon<\varepsilon_{0}$.

REMARK. Notice that the key point of this proof lies in the fact that the preimages $f^{-k p}\left(B_{2^{k} \varepsilon}(a)\right)$ are considered only up to order $k=c-2$. Of course, if we were to take preimages of $B_{2^{k} \varepsilon}(a)$ indefinitely, we would surely end up meeting $\mathcal{C}$ because $B_{2^{k} \varepsilon}(a)$ is a neighbourhood of a point $a \in \mathcal{A} \subset \mathcal{J}(f)$; but, then, also preimages of $\alpha$ would accumulate on $\alpha$ itself so the construction of one such $\mathcal{C}$ would never be possible for a start.

The next step towards the construction of $\mathcal{V}$ is to insure that $\mathcal{U}$ will keep multiple connectivity. This is precisely what the following lemma does.

Lemma 3.5. In the situation described hitherto, there exists $\varepsilon_{1}>0$ such that $f^{-p}\left(V_{\varepsilon}\right)$ has a multiply-connected component in $U$ that separates $E$ and the unbounded connected component of $\widehat{\mathbb{C}} \backslash U$, for all $\varepsilon<\varepsilon_{1}$. 
Proof. Since $U$ is multiply connected, let $\gamma \subset U$ be a generator path of its fundamental group (as a topological space) such that $E$ and the unbounded connected component of $\widehat{\mathbb{C}} \backslash U$ sit in different connected components of $\widehat{\mathbb{C}} \backslash \gamma$ (see Figure 19).



Figure 19: One such generator path $\gamma$, as seen on the Riemann sphere. Notice that it need not separate all the connected components of $\widehat{\mathbb{C}} \backslash U$ pairwise, although it might separate components other than $E$ and the unbounded one.

Consider now the images $\left\{f^{k p}(\gamma)\right\}_{1 \leq k<c}$ in $f^{p}(U)$. Because $\gamma$ accumulates not on points of $\mathcal{J}(f)$, neither do the curves $f^{k p}(\gamma)$ accumulate on points of $\mathcal{A}$, and, therefore, there exist $\left\{\varepsilon_{1, k}>0\right\}_{1 \leq k<c}$ such that, for each $1 \leq k<c, f^{k p}(\gamma) \cap B_{2^{k}}(a)=\emptyset$ for any $\varepsilon<\varepsilon_{1, k}$ and $a \in \mathcal{A}$ (see Figure 20).

In this way, if $\varepsilon<\varepsilon_{1}$, where

$$
\varepsilon_{1}:=\min _{1 \leq k<c} \varepsilon_{1, k}
$$

then $f^{k p}(\gamma) \cap B_{2^{k}}(a)=\emptyset$ for any $1 \leq k<c$ and $a \in \mathcal{A}$. Let us show that it follows from here that $\gamma \subset f^{-p}\left(V_{\varepsilon}\right)$ for all $\varepsilon<\varepsilon_{1}$ : If it were otherwise, $\gamma \nsubseteq f^{-p}\left(V_{\varepsilon}\right)$, then we would have $f^{p}(\gamma) \nsubseteq V_{\varepsilon}$ and, since $f^{p}(\gamma) \subset f^{p}(U)$ and $V_{\varepsilon}=f^{p}(U) \backslash \bigcup_{a \in \mathcal{A}} \bigcup_{k=0}^{c-2} f^{-k p}\left(B_{2^{k} \varepsilon}(a)\right)$, there would exist $0 \leq k \leq c-2$ and $a \in \mathcal{A}$ for which $f^{p}(\gamma) \cap f^{-k p}\left(B_{2^{k} \varepsilon}(a)\right) \neq \emptyset$. So let $z_{0} \in f^{p}(\gamma) \cap f^{-k p}\left(B_{2^{k}}(a)\right)$; taking $f^{k p}$-images we would have $f^{k p}\left(z_{0}\right) \in f^{(k+1) p}(\gamma) \cap B_{2^{k} \varepsilon}(a)$ for some $0 \leq k \leq c-2$, that is, $f^{k p}(\gamma) \cap B_{2^{k} \varepsilon}(a) \neq \emptyset$ for some $1 \leq k<c$, which is in contradiction with the construction of $\varepsilon_{1}$.

Finally, from the fact that $\gamma \subset f^{-p}\left(V_{\varepsilon}\right)$ for all $\varepsilon<\varepsilon_{1}$ and from the choice of $\gamma \subset U$, the lemma follows straightforwardly.

Last, and in a similar spirit to that of the previous lemma, we also want to control the topology of $V_{\varepsilon}$ itself, since it might happen to consist of 


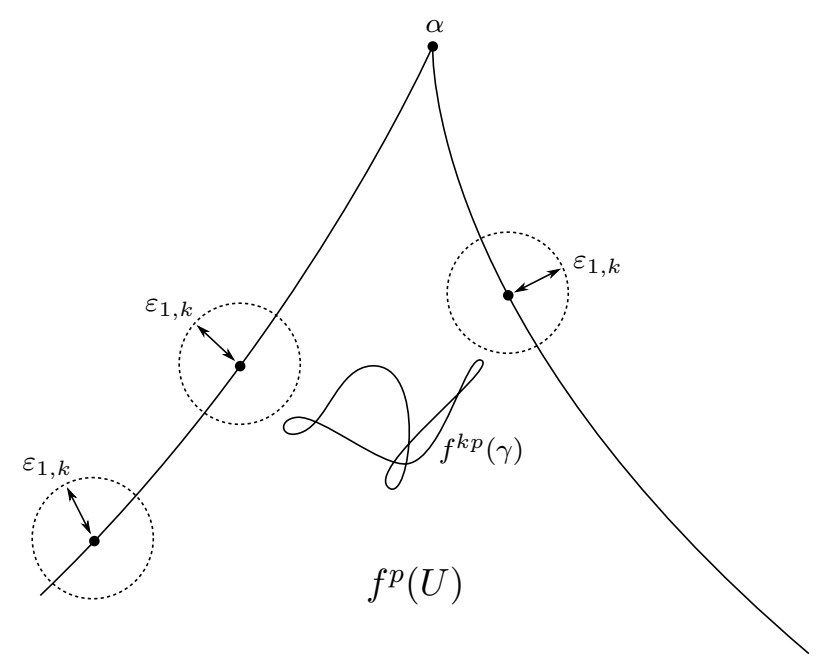

Figure 20: For each $1 \leq k<c$, the radius $\varepsilon_{1, k}$ can be chosen in such a way that $f^{k p}(\gamma) \cap B_{2^{k} \varepsilon}(a)=\emptyset$ for any $\varepsilon<\varepsilon_{1, k}$ and $a \in \mathcal{A}$. Here, the set $\mathcal{A}$ is again represented by the non-labelled points, and we show just one step $1 \leq k<c$ for the sake of clarity.

more than one connected component due to the removal of the preimages $f^{-k p}\left(B_{2^{k} \varepsilon}(a)\right)$ (see Figure 21).

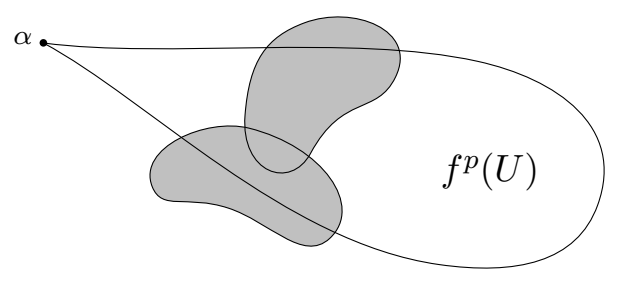

Figure 21: When removing the preimages $f^{-k p}\left(B_{2^{k} \varepsilon}(a)\right)$ (shaded) from $f^{p}(U)$, the resulting set might be disconnected.

This will pose no problem if we focus only on the connected component of $V_{\varepsilon}$ that has $\alpha$ on its boundary, $V_{\varepsilon}^{*}$; but we do have to make sure that the $f^{p}$-preimage of such component will generate a multiply-connected set, as expected.

Lemma 3.6. In the situation described hitherto, there exists $\varepsilon_{2}>0$ such that $f^{-p}\left(V_{\varepsilon}^{*}\right)$ has a component like that of the previous lemma, for all $\varepsilon<\varepsilon_{2}$.

Proof. The construction here is very similar to the proof of Lemma 3.5. In fact, consider $f^{p}(\gamma) \subset f^{p}(U)$, where $\gamma \subset U$ is that path which separates $E$ and the unbounded connected component of $\widehat{\mathbb{C}} \backslash U$. Since $f^{p}(U)$ is simply 
connected and, in particular, path-connected, there exists a (continuous) path

$$
\xi:[0,1] \rightarrow f^{p}(U) \cup\{\alpha\}
$$

such that $\xi(0)=\alpha$ and $\xi(1) \in f^{p}(\gamma)$ (see Figure 22).

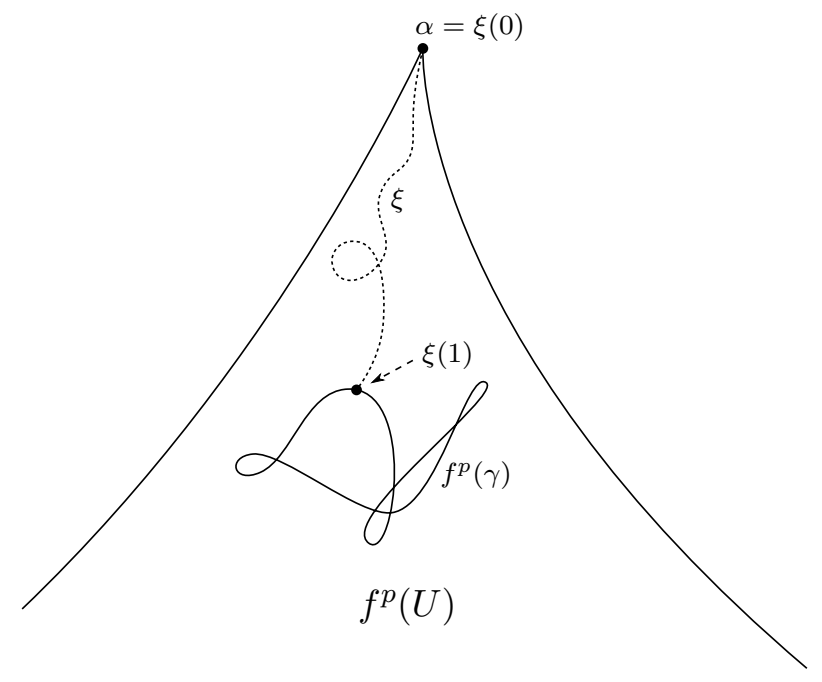

Figure 22: We can connect $\alpha$ and $f^{p}(\gamma)$ by a path $\xi$ in $f^{p}(U) \cup\{\alpha\}$.

Consider now the images $\left\{f^{k p}(\xi)\right\}_{0 \leq k \leq c-2}$ in $f^{p}(U)$. Because $\xi$ accumulates not on points of $\mathcal{J}(f) \backslash\{\alpha\}$, neither do the curves $f^{k p}(\xi)$ accumulate on points of $\mathcal{A}$, and, therefore, there exist $\left\{\varepsilon_{2, k}>0\right\}_{0 \leq k \leq c-2}$ such that, for each $0 \leq k \leq c-2, f^{k p}(\xi) \cap B_{2^{k}}(a)=\emptyset$ for any $\varepsilon<\varepsilon_{2, k}$ and $a \in \mathcal{A}$. In this way, it is clear that if $\varepsilon<\varepsilon_{2}$, where

$$
\varepsilon_{2}:=\min _{0 \leq k \leq c-2} \varepsilon_{2, k},
$$

then $\xi \cap f^{-k p}\left(B_{2^{k}}(a)\right)=\emptyset$ for any $0 \leq k \leq c-2$ and $a \in \mathcal{A}$, that is to say, $\xi \subset V_{\varepsilon}$ and therefore $f^{p}(\gamma) \subset V_{\varepsilon}^{*}$.

Using an identical argument to that of the proof of Lemma 3.5 the result follows.

This completes the construction of the modification of $f^{p}(U)$, since now it just remains to define $\mathcal{V} \subset f^{p}(U)$ as $V_{\varepsilon}^{*}$ for some $\varepsilon<\varepsilon_{0}, \varepsilon_{1}, \varepsilon_{2}$, and $\mathcal{U} \subset U$ as the multiply-connected component of $f^{-p}(\mathcal{V})$ that separates $E$ and the unbounded connected component of $\widehat{\mathbb{C}} \backslash U$ given by Lemma 3.6. 


\section{References}

[1] Lars AHLFors and Lipman Bers, Riemann's mapping theorem for variable metrics, Ann. of Math. (2) $\mathbf{7 2}$ (1960), 385-404.

[2] Walter Bergweiler and Norbert Terglane, Weakly repelling fixpoints and the connectivity of wandering domains, Trans. Amer. Math. Soc. 348 (1996), no. 1, 1-12.

[3] Xavier Buff, Virtually repelling fixed points, Publ. Mat. 47 (2003), no. 1, 195-209.

[4] Adrien Doundy and Xavier Buff, Le théorème d'intégrabilité des structures presque complexes, The Mandelbrot set, theme and variations, London Math. Soc. Lecture Note Ser., vol. 274, Cambridge Univ. Press, Cambridge, 2000, pp. 307-324.

[5] Núria Fagella, Xavier Jarque, and Jordi Taixés, On connectivity of Julia sets of transcendental meromorphic maps and weakly repelling fixed points I, Proc. Lond. Math. Soc. (3) 97 (2008), no. 3, 599-622.

[6] Pierre Fatou, Sur les équations fonctionnelles, Bull. Soc. Math. France 47 (1919), 161-271, and 48 (1920), 33-94 and 208-314.

[7] Hans-Günter MeIER, On the connectedness of the Julia-set for rational functions, preprint no. 4 RWTH Aachen, 1989.

[8] John Milnor, Dynamics in one complex variable, Friedr. Vieweg \& Sohn, Braunschweig, 1999, Introductory lectures.

[9] Feliks Przytycki, Remarks on the simple connectedness of basins of sinks for iterations of rational maps, Dynamical systems and ergodic theory (Warsaw, 1986), Banach Center Publ., vol. 23, PWN, Warsaw, 1989, pp. 229-235.

[10] Mitsuhiro SHISHIKURA, The connectivity of the Julia set and fixed points, Complex dynamics, A K Peters, Wellesley, MA, 2009, pp. 257276.

[11] Dennis Sullivan, Quasiconformal homeomorphisms and dynamics. I. Solution of the Fatou-Julia problem on wandering domains, Ann. of Math. (2) 122 (1985), no. 3, 401-418.

[12] Tan Lei, Branched coverings and cubic Newton maps, Fund. Math. 154 (1997), no. 3, 207-260. 